\title{
SOME INTERPOLATION OPERATORS ON A CURVED DOMAIN
}

\author{
A. BABOŞ
}

Abstract. One of the quite simple procedures for constructing multidimensional approximation operators consist in the composition of univariate approximation operators, using tensor product and boolean sum operators. In this paper, using these type of interpolation operators, constructed for functions defined on a triangle and a square with on curved side, we plot the graphs of the interpolation errors of the corresponding interpolation formulas. Also, we give the maximum interpolation errors for the two test functions.

2010 Mathematics Subject Classification: 41 A05; 41 A36; 65 D05.

Keywords: Approximation operators, triangle, square, curved side, graphs, interpolation errors, test functions.

\section{INTRODUCTION}

Approximation operators on polygonal domains with some curved sides have important applications especially in finite element method for differential equations with given boundary conditions and in computer aided geometric design. Such operators were considered in the papers [8], [7], [6]. Lately, such problems were studied in [2], $[1],[3],[11],[9],[10]$, using interpolation operators.

The aim of this paper is to plot the graphs and to find the maximum of the interpolation errors of the interpolation formula of the Lagrange and Hermite type operators on a triangle with one curved side and, respectively, on a square with one curved side.

\section{INTERPOLATION OPERATORS ON A TRIANGLE}

Let $\tilde{T}_{h}=\left\{(x, y) \in \mathbb{R}^{2} \mid x \geq 0, y \geq 0, x+y \geq h\right\}$ be the standard triangle. In [11] the authors consider a standard triangle, $\tilde{T}_{h}$, having the vertices $V_{1}=(h, 0), V_{2}=(0, h)$ and $V_{3}=(0,0)$, two straight sides $\Gamma_{1}, \Gamma_{2}$, along the coordinate 
axes, and the third side $\Gamma_{3}$ (opposite to the vertex $V_{3}$ ), which is defined by the oneto-one functions $f$ and $g$, where $g$ is the inverse of the function $f$, i.e. $y=f(x)$ and $x=g(y)$, with $f(0)=g(0)=h$ and $F$ a real-valued function defined on $\tilde{T}_{h}$.

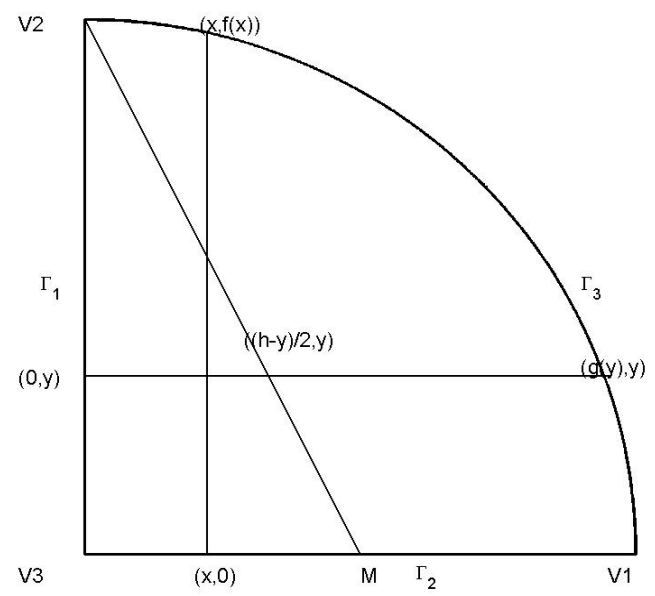

Figure 1: Triangle $\tilde{T}_{h}$

In [11] the authors construct certain Lagrange, Hermite and Birkhoff type operators, which interpolate a given function and some of its derivatives on the border of a triangle with one curved side, as well as some of their product and Boolean sum operators.

In [2] we introduce an Lagrange operator $L_{2}^{x}$ which interpolate the function, $F$, and some interior line of triangle $\tilde{T}_{h}$ and we consider the case when the interior line is a median (Figure 1). $L_{2}^{x}$ interpolate the function $F$ with respect to $x$ in the points $(0, y),\left(\frac{h-y}{2}, y\right),(g(y), y)$ :

$$
\begin{aligned}
\left(L_{2}^{x} F\right)(x, y) & =\frac{(2 x-h+y)[x-g(y)]}{(h-y) g(y)} F(0, y) \\
& +\frac{4 x[x-g(y)]}{(h-y)[h-y-2 g(y)]} F\left(\frac{h-y}{2}, y\right) \\
& +\frac{x(2 x-h+y)}{g(y)[2 g(y)-h+y]} F(g(y), y) .
\end{aligned}
$$


Using this operator and the Hermite type operator from [11]:

$$
\begin{aligned}
\left(H_{2}^{y} F\right)(x, y) & =\frac{[y-f(x)]^{2}}{f^{2}(x)} F(x, 0)+\frac{y[2 f(x)-y]}{f^{2}(x)} F(x, f(x)) \\
& +\frac{y[y-f(x)]}{f(x)} F^{(0,1)}(x, f(x)),
\end{aligned}
$$

we constructe in [1] new interpolation operators.

Let $P$ be

$$
P:=H_{2}^{y} L_{2}^{x}
$$

and

$$
F=P F+R_{1} F
$$

approximation formula generated by $P$, with:

$$
\begin{aligned}
(P F)(x, y) & =\frac{[y-f(x)]^{2}}{f^{2}(x)}\left[\frac{(2 x-h)(x-h)}{h^{2}} F(0,0)-\frac{4 x(x-h)}{h^{2}} F\left(\frac{h}{2}, 0\right)+\right. \\
& \left.+\frac{x(2 x-h)}{h^{2}} F(h, 0)\right] \\
& +\frac{y[2 f(x)-y]}{f^{2}(x)} F(x, f(x))+\frac{y[y-f(x)]}{f(x)} F^{(0,1)}(x, f(x)) .
\end{aligned}
$$

If there exist $F^{(0,1)}$ on the side $\Gamma_{3}$ then $\mathrm{P}$ verifies the interpolation properties:

$$
\begin{aligned}
P F & =F, \text { on } \Gamma_{2} \cup \Gamma_{3} \\
(P F)^{(0,1)} & =F^{(1,0)}, \text { on } \Gamma_{3}
\end{aligned}
$$

and $\operatorname{dex}(P)=2$.

Let $S$ be

$$
S:=H_{2}^{y} \oplus L_{2}^{x}
$$

and

$$
F=S F+R_{2} F
$$

approximation formula generated by $S$, with:

$$
\begin{aligned}
(S F)(x, y) & =\frac{[y-f(x)]^{2}}{f^{2}(x)} F(x, 0)+\frac{(2 x-h+y)[x-g(y)]}{(h-y) g(y)} F(0, y) \\
& +\frac{4 x[x-g(y)]}{(h-y)[h-y-2 g(y)]} F\left(\frac{h-y}{2}, y\right)+\frac{x(2 x-h+y)}{g(y)[2 g(y)-h+y]} F(g(y), y) \\
& -\frac{[y-f(x)]^{2}}{f^{2}(x)}\left[\frac{(2 x-h)(x-h)}{h^{2}} F(0,0)-\frac{4 x(x-h)}{h^{2}} F\left(\frac{h}{2}, 0\right)+\frac{x(2 x-h)}{h^{2}} F(h, 0)\right]
\end{aligned}
$$

Let consider $F: \tilde{T}_{h} \rightarrow \mathbb{R}$ then : 
1. $S F=F$, on $\partial \tilde{T}_{h}$.

2. $\operatorname{dex}(S)=2$.

We consider the following test functions, generally used in literature, (see, e.g., [12]):

$$
\begin{aligned}
& \text { Gentle }: F_{1}(x, y)=\exp \left[-\frac{81}{16}\left((x-0.5)^{2}+(y-0.5)^{2}\right)\right] / 3 \\
& \text { Saddle }: F_{2}(x, y)=\frac{(1.25+\cos 5.4 y)}{6+6(3 x-1)^{2}}
\end{aligned}
$$

In Figure 2- Figure 9 we plot the graphs of the interpolation errors for $L_{2} F_{i}, H_{2} F_{i}$, $P F_{i}$, and $S F_{i}, i=\overline{1,2}$, on $\tilde{T}_{1},(h=1)$ considering $f:[0,1] \rightarrow[0,1], f(x)=\sqrt{1-x^{2}}$.

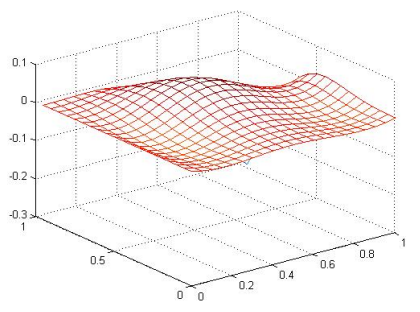

Figure 2: The interpolation error for $L_{2} F_{1}$

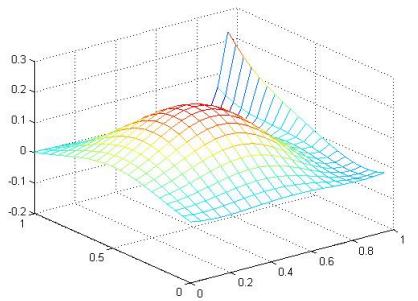

Figure 4: The interpolation error for $P F_{1}$

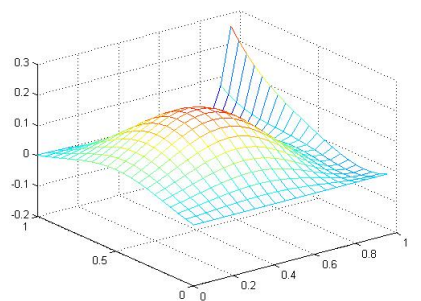

Figure 3: The interpolation error for $\mathrm{H}_{2} \mathrm{~F}_{1}$

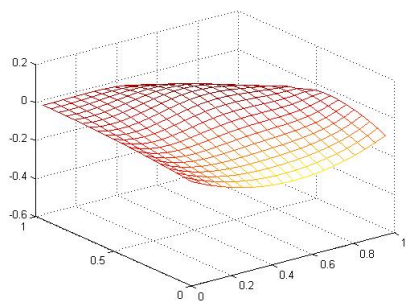

Figure 5: The interpolation error for $S F_{1}$ 


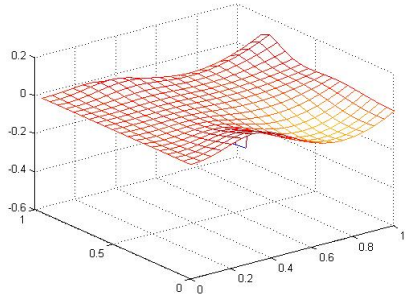

Figure 6: The interpolation error for $L_{2} F_{2}$

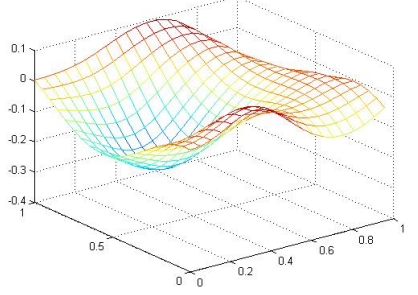

Figure 8: The interpolation error for $P F_{2}$

The following table contains the maximum interpolation errors for the functions given in 7 , defined on $\tilde{T}_{1},(h=1)$.

Table 1: The interpolation error

\begin{tabular}{|l|l|l|}
\hline Max error & $F_{1}$ & $F_{2}$ \\
\hline$L_{2} F$ & 0.0532 & 0.1070 \\
$H_{2} F$ & 0.2554 & 0.0946 \\
$P F$ & 0.2281 & 0.0913 \\
$S F$ & 0.0406 & 0.0297 \\
\hline
\end{tabular}

\section{INTERPOLATION OPERATORS ON A SQUARE}

In [10] the authors consider $D_{h}$ the square with one curved side having the vertices $V_{1}=(0,0), V_{2}=(h, 0), V_{3}=(h, h)$ and $V_{4}=(0, h)$, three straight sides $\Gamma_{1}, \Gamma_{2}$, along 
the coordinate axes, $\Gamma_{3}$ parallel to axis $O x$, and the curved side $\Gamma_{4}$ which is defined by the function $g$, such that $g(h)=g(0)=h$.

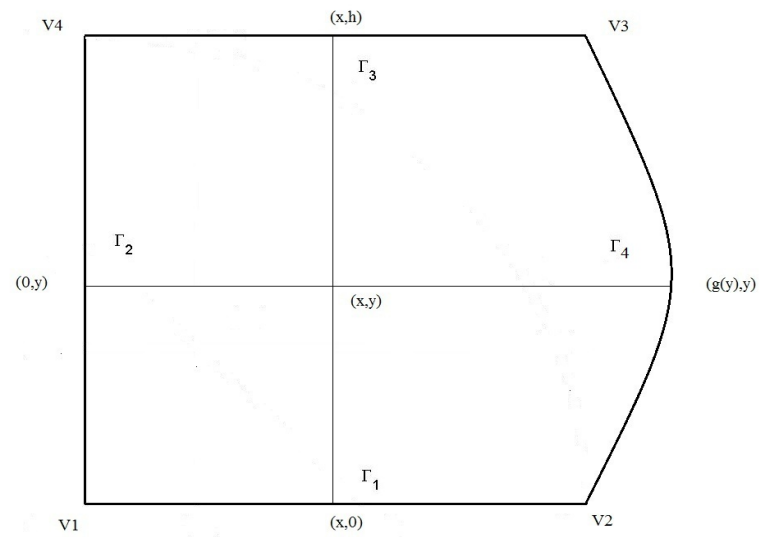

Figure 10: The square $D_{h}$

Let $F$ be a real-valued function defined on $D_{h}$ and $(0, y),(g(y), y)$, respectively, $(x, 0),(x, h)$ be the points in which the parallel lines to the coordinate axes, passing through the point $(x, y) \in D_{h}$, intersect the sides $\Gamma_{2}, \Gamma_{4}$, respectively $\Gamma_{1}$ and $\Gamma_{3}$.

They construct and analyze Bernstein-type operators on the square with one and two curved side.

In [3] we construct the Lagrange operators $L_{1}$ and $L_{2}$ defined by

$$
\begin{aligned}
& \left(L_{1} F\right)(x, y)=\frac{g(y)-x}{g(y)} F(0, y)+\frac{x}{g(y)} F(g(y), y), \\
& \left(L_{2} F\right)(x, y)=\frac{h-y}{h} F(x, 0)+\frac{y}{h} F(x, h) .
\end{aligned}
$$

1) Both operators $L_{1}$ and $L_{2}$ interpolates the function $F$ along two sides of the square $D_{h}$ :

$$
\begin{array}{lll}
\left(L_{1} F\right)(0, y) & =F(0, y) & \left(L_{1} F\right)(g(y), y)=F(g(y), y), y \in[0, h] \\
\left(L_{2} F\right)(x, 0) & =F(x, 0) & \left(L_{2} F\right)(x, h)=F(x, h) \quad x \in[0, h]
\end{array}
$$

2) The degree of exactness $\operatorname{dex}\left(L_{i}\right)=1, \quad i=1,2$. 
Let $P_{21}^{L}$ be the product of the operators $L_{2}$ and $L_{1}$, i.e., $P_{21}=L_{2} L_{1}$.

We have

$$
\begin{aligned}
\left(P_{21^{L}} F\right)(x, y) & =\frac{(h-x)(h-y)}{h^{2}} F(0,0)+\frac{x(h-y)}{h^{2}} F(h, 0) \\
& +\frac{y(h-y)}{h^{2}} F(0, h)+\frac{x y}{h^{2}} F(h, h)
\end{aligned}
$$

1) The interpolation properties: $P_{21}^{L} F=F$, on the four vertices of the square $V_{1}, V_{2}, V_{3}$ and $V_{4}$.

2) The degree of exactness $\operatorname{dex}\left(P_{21}^{L}\right)=1$.

Let $S_{21}^{L}$ be the Boolean sum of the operators $L_{2}$ and $L_{1}$, i.e., $S_{21}^{L}=L_{2} \oplus L_{1}=$ $L_{2}+L_{1}-L_{2} L_{1}$.

We have

$$
\begin{aligned}
\left(S_{21}^{L}\right)(x, y) & =\frac{h-y}{h} F(x, 0)+\frac{y}{h} F(x, h)+\frac{g(y)-x}{g(y)} F(0, y)+ \\
& +\frac{x}{g(y)} F(g(y), y)-\frac{(h-x)(h-y)}{h^{2}} F(0,0) \\
& -\frac{x(h-y)}{h^{2}} F(h, 0)-\frac{y(h-x)}{h^{2}} F(0, h)-\frac{x y}{h^{2}} F(h, h)
\end{aligned}
$$

1) The interpolation properties: $S_{21}^{L} F=F$ on $\partial D_{h}$.

2) The degree of exactness $\operatorname{dex}\left(S_{21}^{L}\right)=1$.

Suppose that the real valued function $F$ is defined on the square $D_{h}$ and it possesses the partial derivatives $F^{(1,0)}$ on the side $\Gamma_{4}$ and $F^{(0,1)}$ on $\Gamma_{3}$.

We consider the operators $H_{1}$ and $H_{2}$ defined by

$$
\begin{aligned}
\left(H_{1} F\right)(x, y) & =\frac{[x-g(y)]^{2}}{g^{2}(y)} F(0, y)+\frac{x[2 g(y)-x]}{g^{2}(y)} F(g(y), y) \\
& +\frac{x[x-g(y)]}{g(y)} F^{(1,0)}(g(y), y), \\
\left(H_{2} F\right)(x, y) & =\frac{(y-h)^{2}}{h^{2}} F(x, 0)+\frac{y(2 h-y)}{h^{2}} F(x, h) \\
& +\frac{y(y-h)}{h} F^{(0,1)}(x, h)
\end{aligned}
$$

1) The interpolation properties:

$$
\begin{aligned}
& \left(H_{1} F\right)=F, \text { on } \Gamma_{2} \cup \Gamma_{4} \quad\left(H_{1} F\right)^{(1,0)}=F^{(1,0)}, \text { on } \Gamma_{4} \\
& \left(H_{2} F\right)=F, \text { on } \Gamma_{1} \cup \Gamma_{3} \quad\left(H_{2} F\right)^{(0,1)}=F^{(0,1)}, \text { on } \Gamma_{3} .
\end{aligned}
$$


2) The degree of exactness $\operatorname{dex}\left(H_{1}\right)=\operatorname{dex}\left(H_{2}\right)=2$

Using the Gentle test function:

Gentle $: F_{1}(x, y)=\exp \left[-\frac{81}{16}\left((x-0.5)^{2}+(y-0.5)^{2}\right)\right] / 3$,

in Figure 10- Figure 13 we plot the graphs of the interpolation errors for $L_{2} F_{1}$, $P_{21} F_{1}, S_{21} F_{i}$ and $H_{2} F_{1}$, on $D_{1},(h=1)$ considering $f:[0,1] \rightarrow[0,1], f(x)=\sqrt{1-x^{2}}$.

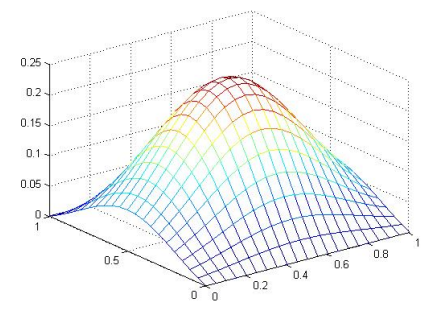

Figure 11: The interpolation error for $L_{2} F_{1}$

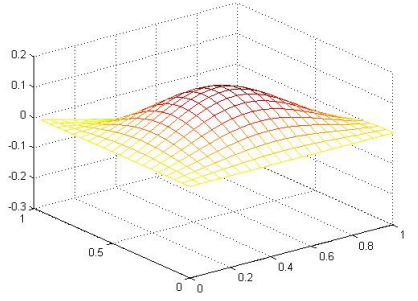

Figure 13: The interpolation error for $S_{21} F_{1}$

The following table contains the maximum interpolation errors for the function given in 8 , defined on $D_{1},(h=1)$.

\section{REFERENCES}

[1] A. Baboş, Some interpolation schemes on a triangle with one curved side, General Mathematics, Vol. 21, No.1-2,(2013), 97-106. 
A. Babos - Interpolation operators ...

Table 2: The interpolation error

\begin{tabular}{|l|l|}
\hline Max error & $F_{1}$ \\
\hline$L_{2} F$ & 0.2393 \\
$P_{21} F$ & 0.3068 \\
$S_{21} F$ & 0.1369 \\
$H_{2}$ & 0.2393 \\
\hline
\end{tabular}

[2] A. Babos, Some interpolation operators on triangle, The 16th International Conference The Knowledge - Based Organization, Applied Technical Sciences and Advanced Military Technologies,Conference Proceedings 3,(2010), 28-34.

[3] A. Babos, Interpolation operators on a square with one curved side General Mathematics Vol. 24, No. 1, (2016), 131-137.

[4] R. E. Barnhill, Representation and approximation of surfaces, Mathematical Software III, J.R. Rice (Ed.), Academic Press, New-York, (1977), 68-119 .

[5] R. E. Barnhill, G. Birkhoff, W. J. Gordon, Smooth interpolation in trian-gles, J. Approx. Theory, 8, (1973), 114-128.

[6] R.E.Barnhill, I.A. Gregory, Polynomial interpolation to boundary data on triangles, Math. Comp., 29 , no. 131, (1975), 726-735.

[7] Barnhill, R.E., Ou, H. S., Surfaces defined on surfaces, Computer Aided Geom. Design, 7 (1990), 323-336.

[8] C. Bernardi, Optimal finite-element interpolation on curved domains, SIAM J. Numer. Anal., 26, no. 5,(1989), 1212-1240 .

[9] P. Blaga, T. Cătinaş, Gh. Coman, Bernstein-type Operators on a Triangle with One Curved Side, Mediterranean Journal of Mathematics, 9(4),(2011), 1-13.

[10] P. Blaga, T. Cătinaş, Gh. Coman, Bernstein-type Operators on a square with one and two curved sides, STUDIA UNIV. BABES BOLYAI, MATHEMATICA, Volume LV, Number 3,(2010), 51-67.

[11] Gh. Coman, T. Cătinaş, Interpolation operators on a triangle with one curved side, BIT Numer Math 47, (2010).

[12] R. J. Renka, A. K. Cline, A triangle-based $C^{1}$ interpolation method, Rocky Mountain J. Math. 14,(1984), 223-237.

[13] M. Zlamal, Curved elements in the finite element method I, SIAM J. Numer. Anal., 10,(1973), 229-240. 
Alina Baboş

Department of Economic Sciences, Faculty of Economic and Administrative Sciences "Nicolae Balcescu" Land Forces Academy,

Sibiu, Romania

email: alina_babos_24@yahoo.com 